\title{
Centaurus A at Ultra-High Energies
}

\author{
Roger W. Clay $^{\mathrm{A}, \mathrm{C}}$, Benjamin J. Whelan ${ }^{\mathrm{A}}$, and Philip G. Edwards ${ }^{\mathrm{B}}$ \\ ${ }^{\text {A }}$ School of Chemistry and Physics, University of Adelaide, Adelaide, SA 5005, Australia \\ ${ }^{B}$ CSIRO ATNF, Narrabri Observatory, Locked Bag 194, Narrabri, NSW 2390, Australia \\ ${ }^{\mathrm{C}}$ Corresponding author. Email: roger.clay@adelaide.edu.au
}

Received 2009 October 19, accepted 2010 December 17

\begin{abstract}
We review the importance of Centaurus A in high-energy astrophysics as a nearby object with many of the properties expected of a major source of very high-energy cosmic rays and gamma rays. We examine observational techniques and the results so far obtained in the energy range from $200 \mathrm{GeV}$ to above $100 \mathrm{EeV}$ and attempt to fit those data to expectations of Centaurus A as an astrophysical source from very high to ultra-high energies.
\end{abstract}

Keywords: acceleration of particles — galaxies: active — gamma rays: observations — cosmic rays

\section{Introduction}

The field of very high-energy astrophysics deals with processes associated with the acceleration and interactions of particles at energies above those accessible by spacecraft observatories, characteristically above a few $100 \mathrm{GeV}$, up to the highest particle energies found in nature, above $100 \mathrm{EeV}$. The massive particles at these energies are known as cosmic rays and at the top of the energy range are referred to as ultra-high-energy (UHE). The observed energetic photons, which are seen at lower energies, are known as very high-energy (VHE) gamma rays.

The all-sky cosmic-ray spectrum exhibits a very steep dependence of flux against energy. It extends over 30 orders of magnitude in flux and ten orders of magnitude in energy to above $100 \mathrm{EeV}$ with rather little deviation from a featureless power law relationship. There is a steepening at $\mathrm{PeV}$ energies, known as the 'knee' (Hillas 1984). At energies in the $\mathrm{EeV}$ range, there is then a flattening known as the 'ankle'. The knee is thought to represent either an energy limit to the acceleration ability of most galactic sources or a limit to the ability of our Milky Way galaxy to securely contain and build up an internal cosmic ray flux. The ankle is thought to represent a change from predominantly galactic sourced cosmic rays to an extragalactic flux (Gaisser \& Stanev 2006). It is thus most reasonable to look at energies above a few EeV for direct observational evidence of Centaurus A (Cen A) as a cosmic-ray source.

The origins of cosmic rays are not securely known. It is thought that supernovae or supernova remnants (SNR) are the most probable origins of cosmic rays that originate in the Milky Way, and that such particles are energized through diffusive shock acceleration (Protheroe \& Clay 2004). There appear to be severe limitations to energies accessible through this process and the highest-energy cosmic rays are postulated to originate in some different environment outside our galaxy (Hillas 1984). Cen A, our closest active galaxy, is a relatively local extragalactic object that contains regions - such as its extended radio lobes or supermassive central black hole - with physical properties which enable cosmic-ray acceleration to exceed the energy limitations that apply in galaxies like the Milky Way. For this reason, over almost 40 years, Cen A has been the target of observational searches for evidence that it is a significant VHE or UHE source. We briefly review the techniques used to study Cen A and the reasons why Cen $\mathrm{A}$ is an attractive observational target, and examine the observational progress which has been made.

\section{Particle Acceleration Processes and Sites}

There is a general expectation that cosmic-ray particles primarily receive energy through diffusive shock acceleration (Berezinskii et al. 1990; Protheroe \& Clay 2004). This is a process whereby charged particles diffusively cross an astrophysical shock front multiple times, receiving a boost in energy with each crossing. This concept has been shown to have the happy characteristic that a power-law cosmic-ray energy spectrum results. Such acceleration processes were first proposed by Fermi, who noted that head-on collisions with moving magnetic clouds resulted in a transfer of energy to already energetic particles, and that head-on collisions were statistically preferred over others (Berezinskii et al. 1990). Fermi's original process proved to be very slow and the realization that multiple (statistical) crossings of a shock front provided a much faster ('first order') process appeared to provide a practical acceleration model. In such a picture, there is a clear requirement that (a subset of) the accelerating particles are repeatedly scattered across the shock. An energy upper limit of the process results from properties of the source region that finally fail to provide sufficient scattering at the highest energies. 
An alternative non-stochastic scenario is that acceleration is associated with the voltage drop created by a rapidly spinning supermassive black hole threaded by magnetic fields induced by currents flowing in a surrounding disk or torus (Levinson 2000). In this case, the maximum achievable energy is apparently in the UHE region, although more detailed modelling will be required to clearly determine limits imposed by energy loss mechanisms such as curvature radiation.

Cen A contains a supermassive black hole and also exhibits evidence of substantial shocks with evidence of particle acceleration associated with their related jets (Hardcastle et al. 2007). We shall see below that it has regions which are capable of scattering particles magnetically as required. Whether those necessary conditions are sufficient for the acceleration of particles to UHE energies is the question to be answered observationally.

\section{Particle Propagation and Attenuation}

At VHE energies, astrophysical particles are capable of having inelastic and elastic collisions with particles and fields in the source, and between the source and our observatories within the Milky Way galaxy. These interactions are important to understanding the astrophysics of the particles which are observed. Although limited in size, source regions can contain strong magnetic fields, intense photon fields over a great energy range, and a high plasma density. In intergalactic space, our knowledge of fields and energy densities is limited but we expect, at least, that there will be some magnetic fields, starlight, infrared radiation, and the cosmic microwave background (CMB; Driver et al. 2008). Closer to home, messenger particles will transit whatever fields are contained in our local group of galaxies, our galactic halo, and the plane of the Milky Way. In the latter context, it is expected that the known magnetic fields of our galaxy (with an underlying regular field at levels of a few $\mu \mathrm{G}$ but up to $10 \mu \mathrm{G}$ if a random component is included; Sun et al. 2008) will have deflected all charged cosmic rays by significant amounts (Stanev 1997). Astrophysical angular uncertainties then exceed instrumental ones for charged cosmic rays.

It is possible for accelerated protons to interact (most likely in a source region) and convert to neutrons. This, for instance, is an argument for a possible cosmic ray excess in the direction of our galactic centre, since the neutrons will not suffer deflection in galactic-plane magnetic fields which would certainly scatter protons out of a directional beam (Clay 2000). Isolated neutrons decay within a few minutes when at rest but cosmic-ray neutrons with a high relativistic gamma factor will survive long distances in the laboratory frame. However, at the distance of Cen A, neutrons with energies below $400 \mathrm{EeV}$ (just above the highest-energy cosmic ray recorded from any direction; Bird et al. 1995) would decay before reaching us. We note that such decay is statistical and some neutrons might be observable even at lower energies but the resulting flux would be greatly attenuated below
$100 \mathrm{EeV}$. It seems that neutrons generated within a Cen A source will not provide a direct undeflected beam, although protons could interact in some intermediate matter, resulting in a 'halo' around the direction of a source.

Cosmic-ray interactions can additionally produce a flux of high-energy neutrinos. These could be through interactions with the $\mathrm{CMB}$ or with particles and fields close to the source. In the latter case, LUNASKA (James et al. 2009) or northern UHE neutrino detectors such as ANTARES (Brown et al. 2009) might search for signals from the direction of Cen A.

\section{Interactions with Photon Fields}

Our Universe is known to be pervaded by the CMB and has a photon number density a thousand times that of characteristic plasma densities. Despite the low energies of microwave photons, VHE photons and UHE nuclei see them as significant targets over modest astrophysical distances. Photons from Cen A are expected to be severely attenuated (Protheroe 1986a,b) over a range of energies, with a spectral cut-off beginning in the energy range $130-200 \mathrm{TeV}$, depending on the strength of the intergalactic magnetic field (Clay et al. 1994). In this absorption feature, attenuation lengths of a few kpc are expected for its deepest point at a little over $1 \mathrm{PeV}$. The absorption feature progressively weakens at higher energies and, for sources within our galaxy, at much higher energies the absorption dip may be passed and photon attenuation may be reduced (Protheroe 1986a). However, for photons from the more distant Cen A with a much greater path length, the absorption will be strong up to $10 \mathrm{EeV}$ (Protheroe 1986b). The Pierre Auger Observatory (PAO; see Section 11) is capable of selecting photons from its overall detected flux at such energies and a search for photons from Cen $\mathrm{A}$ above $10 \mathrm{EeV}$ would seem worthwhile.

Cosmic-ray nuclei will also interact with the CMB but this is not important until energies are much higher than for photons, and the attenuation length is much greater. This attenuation phenomenon is conventionally named the GZK effect after the people (Greisen, Zatsepin and Kuzmin) who proposed it in 1966 for cosmic-ray protons interacting on the CMB (Berezinskii et al. 1990). The interaction has a proton energy threshold of about $60 \mathrm{EeV}$, a factor of 100000 times greater than energies associated with the photon attenuation. There are also interaction processes for other nuclei on the $\mathrm{CMB}$ which become important at about this energy. However, whilst the characteristic attenuation length due to the GZK effect is of the order of $100 \mathrm{Mpc}$, the interaction mean free path is of the order of the distance to Cen A. This is due to the modest energy loss per interaction. Thus both VHE gamma rays and UHE cosmic rays sourced from Cen A are expected to show evidence of interactions with the CMB.

It appears that attenuation compatible with the GZK effect is evident in the all-sky cosmic-ray spectrum of the 
Pierre Auger Observatory (Abraham et al. 2008a), although evidence for a propagation cut-off requires an assumption that the cosmic-ray source spectrum does not contain a similar feature. The current data set at these energies is small and it is questionable if any presently observed events from the direction of Cen A could show statistically convincing evidence for the existence of, or a lack of, GZK attenuation (although see Section 12).

\section{Magnetic Fields}

Charged cosmic-ray particles will have their propagation directions changed in their passage through astrophysical magnetic fields. That deflection will depend on the particle rigidity - the ratio of momentum and charge. At the energies of interest here, this is effectively the ratio of the energy and the charge. A convenient rule of thumb is that the radius of curvature of a $1 \mathrm{PeV}$ proton trajectory perpendicular to a uniform $1 \mu \mathrm{G}$ magnetic field is $1 \mathrm{pc}$. Characteristic galactic fields are at these levels or just above, but their structure and, particularly, their extension out of the galactic plane are poorly known. Also, their strength within our local group of galaxies and the remaining intergalactic space between ourselves and Cen A is largely unconstrained by observation (Beck 2008). There is evidence that richer groups may be pervaded by multiple $\mu \mathrm{G}-$ level magnetic fields (Clarke et al. 2001; Feretti \& Johnston-Hollitt 2004) but this may not apply in our local region. This lack of knowledge is a major problem since, even at proton energies of $100 \mathrm{EeV}$, we are still dealing with a radius of curvature of only $100 \mathrm{kpc}$ in a characteristic magnetic field. As a result, we are unable to specify whether charged cosmic-ray propagation over a distance of $3.8 \mathrm{Mpc}$ from Cen $\mathrm{A}$ is diffusive (with an uncertain magnetic turbulence scale size) or whether we can assume roughly linear propagation. A clear scattered cosmic ray signal from Cen A would give us invaluable information regarding our local extragalactic magnetic fields (though of course this would require a knowledge of the intrinsic source size).

\section{The Air Shower Technique}

Our atmosphere is opaque to primary radiation at the energies with which VHE astrophysics deals. Also, the flux of astronomical particles becomes sufficiently low at those energies and above, such that direct satellite observation ceases to be effective for reasonable spacecraft detector collecting areas. The effective way of working at higher energies is through the use of our atmosphere as a target and observing the cascades of particles, known as 'air showers' or 'extensive air showers' (EAS), which are produced as incident particles deposit their energy, first as conversion to secondaryparticle mass and kinetic energy and then into atmospheric gas excitation and ionization. A good introduction to the physical processes involved in air showers can be found in Allan (1971).

Primary gamma rays initiate cascades which, to a first approximation, develop through successive processes of pair production and then bremsstrahlung of the daughter electrons and positrons. The mean free paths for those processes are related and are between 30 and $40 \mathrm{~g} \mathrm{~cm}^{-2}$ (compared to the thickness of the vertical atmosphere of about $1000 \mathrm{~g} \mathrm{~cm}^{-2}$ ). This rather simple cascade develops 'exponentially' in particle number until ionization energy losses begin to inhibit further development. The cascade thus reaches a maximum particle number (often stated as a number of 'electrons', $N_{e}$ ). Such cascades are statistical in character but the short-interaction mean free paths compared to the total atmospheric depth result in a rather smooth development profile.

Primary nuclei also initiate cascades but these are more complex and irregularly structured. They begin with a strong interaction which produces pions. The neutral pions decay to a pair of gamma rays, which then cascade as we have just seen. However, the charged pions are likely to decay (they may interact again if the atmospheric conditions are conducive) to muons. Those muons will most likely continue to traverse the atmosphere without further major interactions, just suffering a continuous energy loss from their ionization and excitation of atmospheric gases. This cascade now has three components: the remnants of the original particle which only loses a fraction of its initial energy at each interaction (the nuclear core); the muons; and the electromagnetic cascades. A key point is that the nuclear core continues to inject energy through further interactions, resulting in the initiation of superimposed electromagnetic cascades. The overall 'shower' particle number is then a superposition of electron numbers in successive electromagnetic cascades, building and decaying, plus the integrated numbers of muons. This picture is further complicated by the fact that the cosmic-ray beam (at least at the lower energies) is a mixture of nuclear components (Gaisser \& Stanev 2006). These various nuclei have their own interaction mean free paths for initiating cascades (longest for protons, $80 \mathrm{~g} \mathrm{~cm}^{-2}$, and shorter for more massive nuclei) and, though difficult and probably not possible on an event-byevent basis, this offers a means for studying the beam composition, or its change with energy.

All cascades contain charged particles which scatter through interacting with atmospheric gas. As a result, the electromagnetic cascades spread laterally with a characteristic distance of below $100 \mathrm{~m}$ for the numerically dominant electromagnetic component. However, some electromagnetic particles can scatter to very large 'core distances' and ground-based detecting arrays such as the Pierre Auger Observatory record particles at kilometres from a lateral extension of the original cosmic-ray trajectory. The muons scatter rather little but retain their direction from their initiating interaction which results in a characteristic spread of hundreds of metres at sea level. Again, a very few can be found kilometres from the core.

Air shower cascades are studied by sampling a selection of their components. This is efficient in terms of enabling the detection of rare events (at the highest energies the flux may be measured in terms of 
$\mathrm{km}^{-2}$ century $^{-1}$ ). This sampling can be accomplished with a sparse array of ground-based charged particle detectors which sample the cascade at a single development level. The requirement that particles reach the ground limits this technique to energies above $\sim 100 \mathrm{TeV}$ at sea level or to detector arrays located at very high altitudes (Amenomori et al. 2000). Alternatively, that observational energy threshold can be reduced by detecting the bright beam of forward directed Cerenkov light produced in the atmosphere using large optical photon-collecting telescopes which 'image' those photons onto a photomultiplier 'camera'. The VHE gamma-ray telescopes such as the High Energy Stereoscopic System (HESS) (Aharonian et al. 2005) fall into this category. At the highest energies, where a low level of light emission per shower particle is not a limiting factor, isotropic nitrogen fluorescence light, produced by the cascade exciting atmospheric gas, can be successfully recorded. This enables a large collection area to be achieved with large mirrors viewing the cascade from the side. The PAO employs this technique together with a large array of ground-based large-area particle detectors.

\section{Differentiating between Gamma Rays and Cosmic Rays}

In recent years, the study of VHE gamma rays has become an important component of astrophysics. This has become possible partly through improvements in instrumental sensitivity and angular resolution but, also, through significant improvements in the software discrimination between gamma-ray induced EAS and the numerically dominant cosmic-ray showers. The gamma-ray showers are predominantly electromagnetic with interaction processes (pair production and bremsstrahlung) that occur at rather small intervals in the atmosphere. The resulting cascades are rather simple and smooth. On the other hand, cosmic rays initiate and feed cascades through a nuclear process with a much longer mean free path and their interactions produce muons in addition to electromagnetic particles. The cascade development is then rather irregular and also has an irregular geometrical spread due to the muons, which can travel, with rather little scattering, at significant angles away from the central cascade core. Differentiation between gamma-ray and cosmic-ray initiated cascades conventionally depends on vetoing cosmic-ray cascades. This is achieved either by detecting an irregular shower development (or irregular Čerenkov image) - a development which peaks at an atmospheric depth characteristic of nuclei for a given total energy (or particle content) - or a muon content greater than expected for a gamma-ray cascade.

VHE gamma-ray astronomy using the atmospheric Čerenkov technique has been proven to be very efficient in producing images which have good gamma ray to cosmic ray discrimination. This is due to careful Monte Carlo modelling of the cascade and imaging processes to develop suitable image cuts (Aharonian et al. 2005).
These are broadly based on vetoing the larger, less constrained, cosmic ray images, together, in some cases, with a requirement that point sources under study are at known positions in the image.

$\mathrm{PeV}$ gamma-ray studies have more commonly explicitly used a muon veto in which cascades with significant muon numbers have been rejected. This approach has had mixed success. At even higher energies in the EeV range, gamma-ray initiated showers are expected to reach maximum development deeper in the atmosphere than cosmic-ray showers and work is ongoing to select potential gamma-ray cascades on this basis. Presently, the Pierre Auger Observatory claims upper limits to the UHE photon fraction using this method (Abraham et al. 2009).

\section{Searches at TeV Energies}

The first searches for VHE gamma-ray emission from Cen A were made with the Narrabri Stellar Intensity Interferometer. The Intensity Interferometer consisted of two $6.5 \mathrm{~m}$ diameter segmented optical reflectors mounted on a $188 \mathrm{~m}$ diameter circular track. The interferometer took advantage of the Bose-Einstein statistical nature of light, with optical photons from stars tending to arrive in clumps. The correlations in intensity between the two reflectors enabled the diameters of bright stars to be inferred. The light pool from Čerenkov radiation produced by VHE cosmic rays has a similar extent to the track diameter, and so calculations and tests were performed to confirm that the Čerenkov light signal was not contaminating the measurements of steller diameters (Hanbury Brown et al. 1969). It was recognized, however, that the Intensity Interferometer could also be put into service as a Čerenkov detector. Grindlay et al. (1975b) used a $120 \mathrm{~m}$ separation between reflectors and operated the two as a stereo detector. They also employed a novel background rejection scheme, using off-axis photomultipliers to detect Čerenkov light from the penetrating muon component of cosmic-ray initiated cascades, which allowed $\sim 30 \%$ of recorded events to be rejected. Between 1972 and 1974, a sample of 11 sources — including pulsars, X-ray binaries, the Galactic Centre and active galactic nuclei (AGN) - were observed, with source selection based on X-ray and SAS-2 (30 MeV to $100 \mathrm{MeV}$ ) gamma-ray results. A time-averaged $4.5 \sigma$ excess was detected in a total observing time of $51 \mathrm{~h}$ on Cen A (Grindlay et al. 1975a), corresponding to a integral flux above $300 \mathrm{GeV}$ of $(4.4 \pm 1) \times 10^{-11} \mathrm{~cm}^{-2} \mathrm{~s}^{-1}$. As the outer radio lobes were well outside the beam, these were excluded as the source. Possible variability of the gammaray signal (Grindlay et al. 1975a), coupled with theoretical modelling, also excluded the inner radio lobes as the gamma-ray source, with a proposed model of the gammaray flux arising from inverse Compton scattering in the nucleus of Cen A being favoured (Grindlay 1975).

Subsequent VHE observations over the next 35 years yielded negative results. The Durham group, based at Narrabri, observed between 1987 March and 1988 April 
with their MkIII telescope for a total of $44 \mathrm{~h}$ of good onsource data. The $3 \sigma$ flux upper limit above $300 \mathrm{GeV}$ was $7.8 \times 10^{-11} \mathrm{~cm}^{-2} \mathrm{~s}^{-1}$ (Carramiñana et al. 1990). In March $1997,6.75$ h of observations were made with the Durham Mk6 telescope, which provided better discrimination against the cosmic-ray background, with a $3 \sigma$ flux upper limit above $300 \mathrm{GeV}$ of $5.2 \times 10^{-11} \mathrm{~cm}^{-2} \mathrm{~s}^{-1}$ (Chadwick et al. 1999).

The JANZOS group observed Cen A from New Zealand for $56.9 \mathrm{~h}$ between April 1988 and June 1989, reporting a $95 \%$ confidence level upper limit on the flux above $1 \mathrm{TeV}$ of $2.2 \times 10^{-11} \mathrm{~cm}^{-2} \mathrm{~s}^{-1}$ (Allen et al. 1993a).

Interest in VHE emission from Cen A was rekindled by the EGRET detection of Cen $\mathrm{A}$ in the $30 \mathrm{MeV}$ to $30 \mathrm{GeV}$ range (Steinle et al. 1998; Sreekumar et al. 1999). The CANGAROO $3.8 \mathrm{~m}$ telescope was used in 1999 March and April to record a total of $45 \mathrm{~h}$ of on- and off-source data. The resulting $3 \sigma$ flux upper limits above $1.5 \mathrm{TeV}$ were $5.5 \times 10^{-12} \mathrm{~cm}^{-2} \mathrm{~s}^{-1}$ for a point source at the core of the galaxy, and $1.3 \times 10^{-11} \mathrm{~cm}^{-2} \mathrm{~s}^{-1}$ for an extended region of radius 14 arcmin centred on the core (Rowell et al. 1999). In 2004 March and April, further observations were made with three $10 \mathrm{~m}$ telescopes of the CANGAROO-III array. From $10.6 \mathrm{~h}$ of on-source data, upper limits were set for the several regions of interest: for the core of Cen A the $2 \sigma$ upper limit above $424 \mathrm{GeV}$ was $4.9 \times 10^{-12} \mathrm{~cm}^{-2} \mathrm{~s}^{-1}$ (Kabuki et al. 2007).

The HESS group achieved a detection of Cen A with over $120 \mathrm{~h}$ of observation between 2004 April and 2008 July. Their measured integral flux above $250 \mathrm{GeV}$ was $(1.56 \pm 0.67) \times 10^{-12} \mathrm{~cm}^{-2} \mathrm{~s}^{-1}$. The detection is concentrated on the central galaxy region, not the lobes. This excess 'only matches the position of the core, the $\mathrm{pc} / \mathrm{kpc}$ inner jets and the inner radio lobes' (Aharonian et al. 2009).

The HESS flux is a factor of almost 30 below the original report of Grindlay et al. (1975a). However, the Intensity Interferometer observations were made during an extended period of enhanced X-ray emission in the early 1970s. Although X-ray monitoring was infrequent during the 1980s, it appears Cen A has been in a relatively quiescent state for most of the last 30 years (Bond et al. 1996; Turner et al. 1997; Steinle 2006). A low X-ray state would plausibly result in a low flux of inverse Compton scattered VHE gamma rays.

$\mathrm{TeV}$ detections and upper limits are plotted in Figure 1.

\section{Searches at PeV Energies}

Searches at $\mathrm{PeV}$ energies are made using air shower arrays of particle detectors. The Buckland Park air shower array was used between 1978 and 1981 to study the anisotropy of cosmic rays above an energy of $1 \mathrm{PeV}$. The directional accuracy of the array was $3 \operatorname{deg} \times \sec (\theta)$, where $\theta$ is the zenith angle. The study confirmed that the overall cosmic-ray flux shows no strong sidereal isotropy at these energies, and the work was then extended to search for more localized excesses. The least isotropic

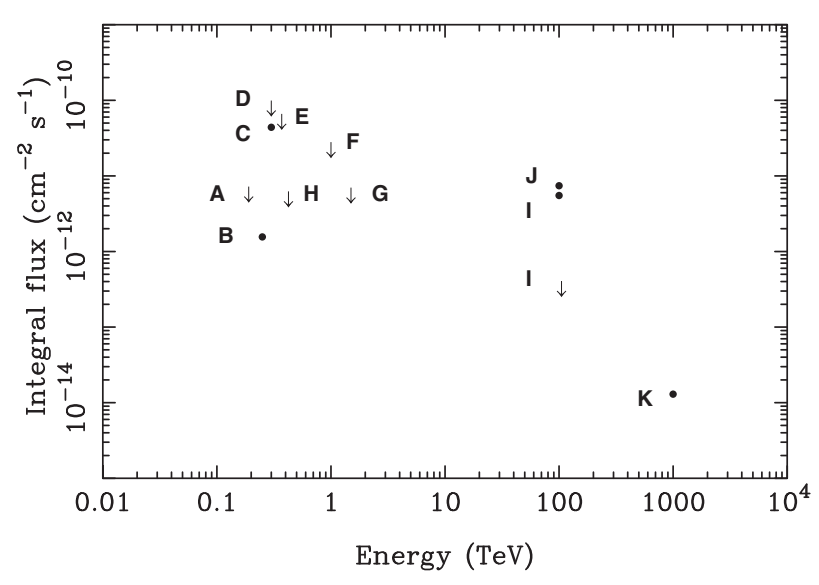

Figure 1 Reported detections (solid circles) and upper limits (arrows) to the flux from $\mathrm{Cen} \mathrm{A}$ at $\mathrm{TeV}$ and $\mathrm{PeV}$ energies. Labels are as follows: A, HESS, Aharonian et al. (2005); B, HESS, Aharonian et al. (2009); C, Narrabri, Grindlay et al. (1975a); D, Durham, Carraminana et al. (1990); E, Durham, Chadwick et al. (1999); F, JANZOS, Allen et al. (1993a); G, CANGAROO, Rowell et al. (1999); H, CANGAROO-III, Kabuki et al. (2007); I, JANZOS, Allen et al. (1993b); J, Buckland Park, Clay et al. (1994); K, Buckland Park, Clay et al. (1984). The Chadwick et al. (1999) point (E) has been moved horizontally from the actual $300 \mathrm{GeV}$ energy threshold for clarity.

declination band was that between $-40^{\circ}$ and $-45^{\circ}$, and the largest excess in bins of $1 \mathrm{~h}$ in right ascension coincided with Cen A. The overall significance was $2.7 \sigma$, a value not unexpected by chance given the number of bins examined, but which encouraged further investigation. There was some evidence in the binned data for excess event numbers toward both outer radio lobes (Clay et al. 1984). Some supporting evidence was also noted in two other Southern Hemisphere experiments (Farley and Storey 1954; Kamata et al. 1968), though with differing energy thresholds, angular resolutions, and years of operation. It was also acknowledged that, at $\mathrm{PeV}$ energies, the signal could not be due to gamma rays from Cen A as the path length for interactions of $\mathrm{PeV}$ gamma rays with CMB photons is only $10 \mathrm{kpc}$ (Clay et al. 1984).

The JANZOS experiment combined $\mathrm{TeV}$ telescopes with a PeV scintillator array, and a search for gamma rays above $100 \mathrm{TeV}$ was conducted with data taken between 1987 October and 1992 January. No significant excess was found over this complete time range, but an excess was observed over 48 d from 1990 April to June, with the excess concentrated in events with energies below $200 \mathrm{TeV}$, consistent with the effects of the expected absorption at higher energies. Monte Carlo simulations were used to derive a probability of $2 \%$ for the observed $3.8 \sigma$ excess to arise by chance (Allen et al. 1993b). A contour map of the significance showed a peak that coincided, within the angular resolution of the array, with the core of Cen A. Buckland Park data taken between 1988 March and 1989 February was examined and no significant excess found from Cen A (Bird \& Clay 1990) — this being consistent with the JANZOS result for this period. 
A larger Buckland Park data set, from 1984 to 1989 , was split a priori into three event size bins, and an excess found in the lowest size bin, corresponding to energies below $150 \mathrm{TeV}$ (hence below the CMB absorption feature; Clay et al. 1994). A confidence level of $99.4 \%$ was claimed for the excess. A Kolmogorov-Smirnov test indicated there was no significant evidence for enhanced periods of emission over this five-year period. A contour map of the excess showed a peak suggestive of a point source compatible with the core of Cen A.

$\mathrm{PeV}$ detections and upper limits are also plotted in Figure 1.

\section{Sydney University Giant Air Shower Recorder}

The Sydney University Giant Air Shower Recorder (SUGAR; Winn et al. 1986), apart from having a creative acronym, was notable and important in pioneering the start of a new era of cosmic ray study. Like the huge PAO (Section 11), with an enclosed area of $70 \mathrm{~km}^{2}$, SUGAR's design recognized that the flux of cosmic rays at the highest energies is so low that its ground detectors required large separations and could not realistically be connected by cable to their direct neighbours. They required a measure of local autonomy. In the case of SUGAR, this was through the realization of a local coincidence between two muon detectors at each station and the tape recording of their data plus time stamping for later global array analysis. The detector sites were also autonomous in terms of power, generating their own power thermoelectrically. Previous arrays had detected real time coincidences between spaced detectors to initiate data acquisition following the arrival of a suitably energetic shower. SUGAR operated for long enough to detect a significant number of showers with energies above the GZK cut-off energy and, until the commissioning of the PAO, was the only array at the highest energies with Cen A in its field of view. SUGAR was shown to have a problem with photomultiplier afterpulsing which could make some energy assignments uncertain, although its direction determinations would not have been affected by that. Data from this array was used for studying a possible association of the highest-energy cosmic rays with the direction of our galactic centre (Bellido et al. 2001). No excess from the direction of Cen A was evident in that analysis. Figure 2 shows the SUGAR highest-energy events (SUGAR Catalogue 1986 ) in the vicinity of Cen A (for comparison with Figure 3 for the PAO).

\section{The Pierre Auger Observatory}

The Pierre Auger Observatory (PAO) is the largest cosmic-ray detector ever built. It currently consists of a Southern Hemisphere site located near the town of Malargüe, Argentina, with planning of a Northern Hemisphere site in Colorado, USA, underway (Harton et al. 2009). The southern site covers an area of approximately $3000 \mathrm{~km}^{2}$ (Suomijärvi et al. 2009). The

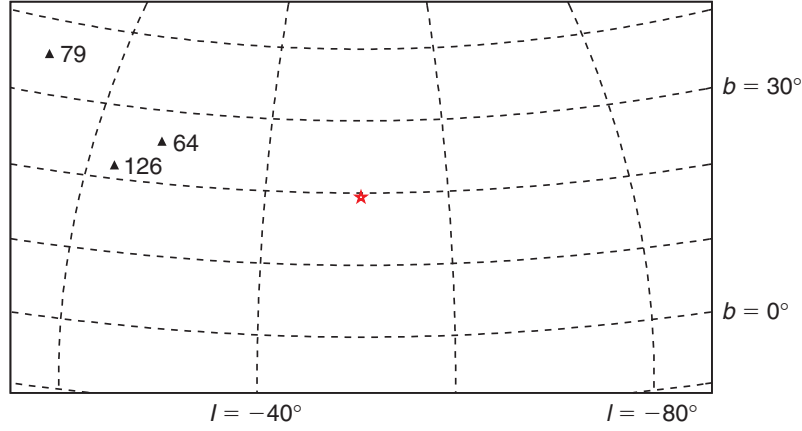

Figure 2 SUGAR events with energies above $60 \mathrm{EeV}$ in the vicinity of Cen A (SUGAR Catalogue 1986). The events are labelled by their energy in EeV. The red star indicates the position of Cen $\mathrm{A}$.

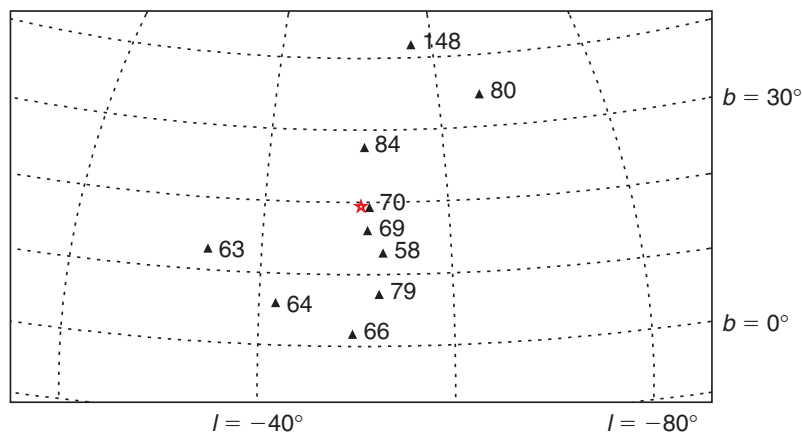

Figure 3 Auger events above $57 \mathrm{EeV}$ in the vicinity of Cen A (Abraham et al. 2008b). The events are labelled by their energy in $\mathrm{EeV}$. The red star indicates the position of Cen A.

PAO employs two cosmic-ray detection methods through the surface detector (SD) and the fluorescence detector (FD; Abraham et al. 2004; Bellido et al. 2005; Allekotte et al. 2008).

The SD consists of more than 1600 autonomous particle detector stations which employ the waterCerenkov detection technique. The particle detectors operate by recording Čerenkov light emitted when relativistic charged particles in an air shower pass through $1.2 \mathrm{~m}$ deep purified water enclosed in large-area $\left(10 \mathrm{~m}^{2}\right)$ tanks. These stations are arranged on a triangular grid, with $1.5 \mathrm{~km}$ spacing. This spacing results in the SD being fully efficient for detecting showers with a primary energy of above $3 \times 10^{18} \mathrm{EeV}$ at zenith angles of $60^{\circ}$ or less (Suomijärvi et al. 2009). Statistical energy uncertainties from the SD are approximately $17 \%$, with an additional systematic uncertainty of $7 \%$ at $10^{19} \mathrm{eV}$ (increasing to $15 \%$ at $10^{20} \mathrm{eV}$ ) arising from calibration with FD energies (Di Guilio et al. 2009). Directional uncertainties are approximately $1.5 \mathrm{deg}$ at energies around $3 \mathrm{EeV}$, reducing to less than $1 \mathrm{deg}$ for energies above approximately $10 \mathrm{EeV}$ (Bonifazi et al. 2009). It has a duty cycle of slightly less than $100 \%$ giving a current integrated exposure of more than $12000 \mathrm{~km}^{2} \mathrm{sryr}$, increasing by $\sim 350 \mathrm{~km}^{2}$ sr yr per month (Schüssler et al. 2009).

The FD makes use of the air fluorescence method. Twenty-four telescopes are separated into four groups of 
six telescopes (each group being termed a FD 'site'), which overlook different sections of the SD. Each telescope views $30 \mathrm{deg}$ in azimuth, giving each site a $180 \mathrm{deg}$ azimuthal field of view, and $28.6 \mathrm{deg}$ in elevation. In each telescope a camera consisting of an array of photomultiplier tubes, viewing separate regions of sky, collects the light emitted by nitrogen molecules excited by the EAS. Using pulse timing information from triggered pixels, the axis along which the shower front propagates can be reconstructed. The energy of the shower, apart from a small amount of 'invisible energy' carried by neutrinos and muons, which are not visible to the FD, is proportional to the integrated light flux along the shower's path. This allows an effectively calorimetric measurement of particle energy (Bellido et al. 2005). The statistical energy uncertainties are approximately $9 \%$, with a systematic uncertainty of approximately $22 \%$ arising from factors such as limitations of knowledge of the instantaneous atmospheric profile and aerosol content (Di Guilio et al. 2009). The requirement of clear, moonless nights for the operation of the FD means that its duty cycle is about $13 \%$ (Schüssler et al. 2009).

The colocation of the FD and SD allows events to be observed by both detectors. The events for which this occurs are termed 'hybrids'. For these events, the timing information from a triggered SD station is added to that from the FD trace to reconstruct the arrival direction (Bellido et al. 2005). This allows a greater accuracy in determining the shower axis than is possible with either method alone, and the average hybrid directional uncertainty is $0.6 \mathrm{deg}$ (Bonifazi et al. 2009). An additional advantage of the hybrid method is that it allows the SD energy scale to be determined. By a comparison of independent SD and FD reconstructions of the same events, SD energies can be calibrated against the essentially calorimetric values from the FD (Di Guilio et al. 2009).

Composition studies are performed primarily with the FD through measurements of the position of shower maximum, $X_{\max }$. This value indicates the slant depth in the atmosphere, in $\mathrm{g} \mathrm{cm}^{-2}$, at which the flux of fluorescent light from the EAS reaches its maximum. From shower to shower, the value of $X_{\max }$ fluctuates due to the statistical nature of shower initiation and development. On average, however, nuclei are expected to have smaller $X_{\max }$ values than protons at a given energy, and fluctuations in $X_{\max }$ are expected to be smaller. Consequently, FD measurements are used to study the behaviour, as a function of energy, of both $\left\langle X_{\max }\right\rangle$, the energy dependence of which is termed the 'elongation rate', and the root mean square (RMS) of $X_{\max }$ to look for possible changes in the composition of the primary particles (Bellido et al. 2009). It should be noted, however, that the interpretation of these results is not clear due to uncertainties in hadronic interaction physics at such high energies (Bellido et al. 2009; D'Urso et al. 2009).

The large duty cycle of the SD makes the prospect of utilizing it to determine cosmic-ray composition highly desirable. This is a somewhat more difficult task than with the FD, however, as a direct measurement of $X_{\max }$ is not possible with the SD. Methods such as studying the risetime of the particle signal in the SD stations, the shower front radius of curvature, the ratio of the muonic to electromagnetic contributions to the signal, and the azimuthal asymmetry in station signal around the shower axis are currently being investigated for their suitability in composition determination with the SD (Wahlberg et al. 2009).

\section{Observational Results at the Highest Energies}

The PAO is the only system presently recording data at EeV energies from the direction of Cen A. It presented its first sky map in 2007 (Abraham et al. 2007) displaying the 27 highest-energy events at that time. This map appears to show event clustering in the general direction of Cen A. Data in that sky map that are in the vicinity of Cen A are shown in Figure 3 (Abraham et al. 2008b), which illustrates apparent clustering around the direction of Cen A.

The Auger paper interprets the data set as a whole as being statistically associated with the directions of local AGN. This was on the basis of an a priori search 'prescription'. The Pierre Auger Collaboration has not yet developed a discovery prescription for Cen A and, as a result, no a priori statistical analysis is presently possible. However, one can comment on the properties of the data set.

Hillas (2009) has independently examined this Auger data set and confirms the conclusion that the highest energy events are associated with rather typical Seyfert galaxies in clusters at distances of typically $\sim 50 \mathrm{Mpc}$. The clustering of events near Cen A is confirmed and an origin in Cen A, or alternatively, NGC 5090 considered. The close proximity of Cen A, however, led Hillas to conclude that Cen $\mathrm{A}$ is probably an inactive $10^{20} \mathrm{eV}$ accelerator as more distant galaxies play a larger role than might have been expected on the basis of a simple inverse square law flux dependence.

More recently, (Hague et al. 2009), the Pierre Auger Collaboration has shown continuing evidence for a concentration of the highest energy events in the direction of Cen A. Those data show an excess of events above $55 \mathrm{EeV}$ within $18 \mathrm{deg}$ of Cen A, which is well above the $68 \%$ confidence interval for a sample from an isotropic distribution. In that range, 12 events are found, where 2.7 are expected on the basis of an isotropic flux. Of the Auger events, $\sim 30 \%$ show some evidence of being members of such clustering out to $30 \mathrm{deg}$ from Cen A, approximately the same fraction $(10 / 27)$ as found in the original Auger sky map.

On the basis of this evidence, one might speculate that Cen $\mathrm{A}$ is the source of a substantial fraction of the extragalactic cosmic rays at energies above the ankle of the cosmic-ray energy spectrum. In that case, one notes that the angular size of the cosmic ray 'image' is appreciably greater than the known physical dimensions of the astronomical source on the sky. That cannot be explained 
by instrumental errors since, as noted above, the known angular resolution of the observatory at these energies is below 1 deg (Bonifazi et al. 2009). One naturally invokes magnetic scattering in intergalactic space or within our galactic region. If the scattering occurs in intergalactic space, one might plausibly assume a $10 \mathrm{kpc}$ turbulence cell size, leading to a total scattering deflection of the order of 20 times the scattering in an individual cell (after the passage of approximately 400 individual cells). To fit the observed excess around Cen $\mathrm{A}$, this requires a turbulent intergalactic field of strength $0.1 \mu \mathrm{G}$.

At these energies, any deflections from the direction of Cen A in known regular galactic plane magnetic fields are likely to be modest (a few degrees; Stanev 1997) but the extent and strength of magnetic fields in any galactic halo around the Milky Way are unknown (Sun et al. 2008) and could plausibly be substantial if the dimensions of the halo are large. The Auger excess appears to limit the possibility of such effects from regular fields since there seems to be no great asymmetry perpendicular to the galactic plane although there may be an oval axis in that direction for the excess just discussed.

In a scenario in which the object Cen $\mathrm{A}$ is the origin of the Pierre Auger 'Centaurus A excess', one must explain the apparent similarity between the distribution of energies within the excess when compared with the totality of Auger events. Using the published event data (Abraham et al. 2008b), there is no significant difference between the two spectra (Figure 4) on the basis of a KolmogorovSmirnov test (at $>99 \%$ level), admittedly with a very limited event list. If that continues to be the case, one might have to abandon the GZK cut-off as the source of a deficit of events above $60 \mathrm{EeV}$ and argue for a source acceleration limitation.

An examination of event data presented by the Pierre Auger Collaboration with their 2007 sky map of the highest-energy events shows a possible systematic variation in particle energy across the Cen A excess, with the highest energy events being at the highest (positive) galactic latitudes. Such an effect could be due to chance,

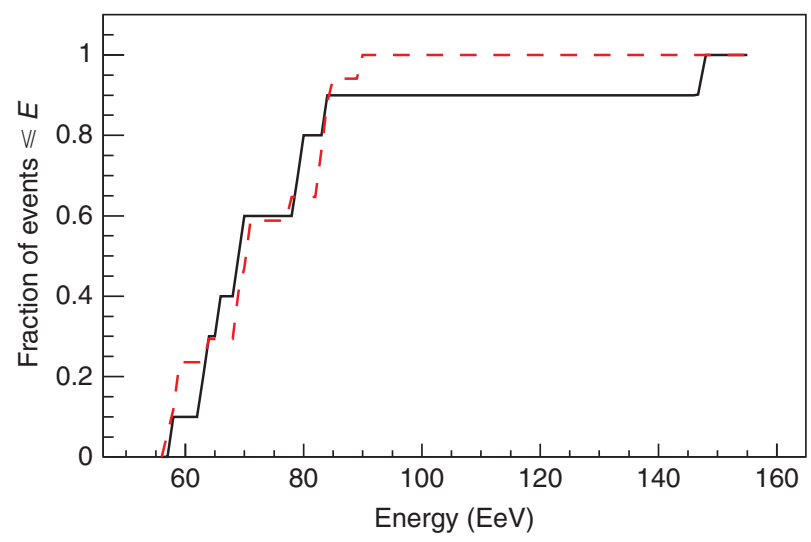

Figure 4 Energy distribution for events inside and outside a $25 \mathrm{deg}$ circle centred on Cen A (Abraham et al. 2008b). The dotted red line is for the events more than $25 \mathrm{deg}$ from Cen A. but it could also count as evidence against the excess being associated with Cen A since there is not now a symmetry in the event energies about the central region. An explanation could be that the cosmic-ray source for the highest energy events is in the northern lobe, or there could be contamination from another source in the supergalactic plane, or simply that the combination of intergalactic scattering and the passage through structured regular fields combine to produce the effect.

The energy variation with direction could also suggest that there is another possible approach to understanding the Cen A excess. This is that the propagation is dominated by regular intergalactic fields and that the particles are deflected as in a magnetic spectrometer, with the true source being in the direction from which an 'infinite' energy particle would have been seen. Since the excess has its highest energy particles furthest north from the galactic plane, one would invoke a magnetic field parallel to that plane (perpendicular to the supergalactic plane) with a 'true' source region some distance further to the north such that the angular deflection is inversely proportional to the particle rigidity. In this scenario, it could be that the deflecting magnetic field is a halo field of the Milky Way. This would require a product of magnetic field strength for the regular component of the field and its spatial extent of the order of $50 \mu \mathrm{G} \mathrm{kpc}$. This may not be incompatible with models of magnetic fields in groups of galaxies or extended galactic halos.

\section{Centaurus A as a Cosmic-Ray Source}

As we saw, it is usual to think of cosmic rays being accelerated to their observed energy in a rather slow statistical process. There are alternative possibilities, such as a single acceleration through a very large potential step or a 'top down' model in which an ultra-energetic particle is the result of the decay of an exotic highly massive initial particle. If the process is something like diffusive shock acceleration, the acceleration site must be such that its scattering fields are capable of returning accelerating particles many times across a shock front. This would appear to require local magnetic fields with products of strengths and physical dimensions such that a particle radius of gyration at the highest energies can be contained within the physical boundaries of the field. This is often expressed through one of the 'Hillas diagrams' (Hillas 1984).

When it comes to considering Cen A as a source, possible extremes of the spectrum of sites would be within the modest strength magnetic fields enclosed in one or other of its giant radio lobes, or (at the other extreme) within very strong fields close to the central engine. Somewhere within a jet, or the southern shock, could also be candidate sites specific to $\mathrm{Cen} A$. The radius of gyration of a cosmic-ray proton (in $\mathrm{kpc}$ ) is numerically close to its energy (in units of EeV) divided by the magnetic field strength (in units of $\mu \mathrm{G}$ ). Containment within an acceleration region will require that the region is 
significantly larger than that radius of gyration. Since it is the particle rigidity which is relevant, this requirement would be eased in proportion to the nuclear charge for heavier nuclei. This would mean that the acceleration of protons in $200 \mathrm{kpc}$ lobes of Cen A (the whole of a lobe) would require magnetic fields filling a lobe at microgauss levels in order to accelerate particles to the measured Auger limit of about $200 \mathrm{EeV}$.

Under such conditions (Protheroe \& Clay 2004 equation 41), a time of the order of 100 million years is required for the acceleration process. This would seem to be approximately the limit of possible acceleration both under a $10^{8}$ year estimate of AGN lifetimes and an estimate of $\mu \mathrm{G}$ strength fields in the lobes. The cosmic-ray energy spectrum extends over 30 orders of magnitude in flux and very few accelerated cosmic rays are required to reach the highest energies - they are statistical anomalies. The source magnetic field must be strong enough and large enough in scale for the highest energy particles to be capable of one last diffusive scattering across the shock front.

An alternative extreme of the spectrum of possible Cen A acceleration sites might be within the most central volume of the AGN, close to the black hole. The majority of $\mathrm{TeV}$ and $\mathrm{PeV}$ detections are consistent with an excess concentrated on the central galaxy region, and not the outer lobes. A central region with dimensions of, say, $10 \mathrm{pc}$ would require a shock containment field strength approaching $1 \mathrm{G}$. This would be substantial but not unreasonable. A difficulty with such a region would be to accelerate particles to UHE energies over a substantial period of time within a dense photon field containing photons with energies substantially above those of the CMB. This is because cosmic ray energy loss interactions would be significant from at least EeV energies. This attenuation at energies below the ankle of the cosmic-ray spectrum makes it difficult to see how the Auger Cen A spectrum could bear similarity with the conventional spectrum from other directions.

Cen $\mathrm{A}$ is a radio galaxy with highly extended jets and lobes. As we noted, it could be that such lobes play a key part in accelerating particles to the highest observed energies. Nagar \& Matulich (2008) have discussed the possible role of objects with that morphology as sources of the Auger highest-energy events. They note that there is a number of such sources (5) in the general vicinity of Cen A out of a total of 10 in the 'field of view' of the PAO. They also note that such objects seem to be statistically closely related to the directions of the highest energy PAO events. This proposition seems to be arguable but, if this is the case, the contribution of Cen A to the total flux must be below a level proportional to its radio emission since, including all such objects, Cen A dominates the total sum of the radio fluxes typically by at least an order of magnitude (Nagar \& Matulich 2008). This is due to its proximity to us, and it could be that some of the other objects are more effective at accelerating particles to the highest energies. We noted that Cen A may be a variable source at high energies, which may support this idea (Hillas 2009).

Recently, Rieger \& Aharonian (2009) have considered Cen A as a VHE gamma-ray and UHE cosmic-ray source, and conclude that advection dominated accretion disk models can account for the production of the TeV emission close to the core via inverse Compton scattering of sub-mm disk photons by accelerated electrons. As it is unlikely that protons could be accelerated to $\mathrm{EeV}$ energies in this region, they propose shear acceleration along the kpc-scale jet as the origin for these particles.

\section{Conclusions}

Centaurus A has been a popular potential source of cosmic rays for close to half a century. A number of cosmic ray and VHE gamma ray searches for excesses from that region have been made. An early search by Grindlay et al. (1975a,b) with VHE gamma rays was encouraging, showing evidence for a positive observation at a time of a large X-ray flare, and, very recently, HESS has also provided evidence that Cen A is a VHE gamma-ray source. The Buckland Park air shower array found a signal with appropriate spectral characteristics which included evidence for CMB absorption at sub-PeV energies. Also, recently, the PAO has shown evidence of a clustering of UHE cosmic-ray events around Cen A although without evidence for a different spectrum to that of other directions. Taken with observations which have produced upper limits and recognizing that none of these observations had well defined a priori statistical analysis procedures, one cannot say with confidence that Cen $\mathrm{A}$ is a major source at UHE energies. However, it is the nearest example to us of one of the few classes of objects which have been identified as having a structure possibly capable of accelerating particles to the highest observed energies. Acceleration of cosmic-ray particles to the highest measured energies in Cen A would be at the limit of parameters associated with the acceleration process. A reduction in the cosmic-ray flux (from the general direction of Cen $\mathrm{A}$ ) above $\sim 60 \mathrm{EeV}$ from the power law at lower energies could be a source effect rather than a GZK cut-off, which would (and may well) otherwise apply to more distant sources.

For the future, we clearly require more data. Further work at VHE (HESS) energies to better define the source region and any possible extended structure would be great progress. An extension of the gamma-ray spectrum upwards towards the CMB absorption feature using the Cerenkov technique, with better angular uncertainty than Buckland Park, such as the TenTen concept (Rowell et al. 2008), would be a major asset. Also, solid confirmation and understanding of the Pierre Auger 'Centaurus A excess' is urgently needed.

\section{References}

Abraham, J. et al. (Pierre Auger Collaboration), 2004, Nucl. Instr. Meth. Phys. Res. A, 523, 50

Abraham, J. et al., 2007, Science, 318, 938 
Abraham, J. et al., 2008a, Phys. Rev. Lett., 101, 061101

Abraham, J. et al. (Pierre Auger Collaboration), 2008b, Astropart. Phys., 29, 188

Abraham, J. et al., 2009, Astropart. Phys., 31, 399

Aharonian, F. et al. (HESS Collaboration), 2005, A\&A, 442, 177

Aharonian, F. et al., 2009, ApJ, 695, L40

Allan, H. R., 1971, Prog. Elem. Part. Cos. Ray Phys., 10, 171

Allekotte, I. et al. (Pierre Auger Collaboration), 2008, Nucl. Instr. Meth. Phys. Res. A, 586, 409

Allen, W. H. et al., 1993a, ApJ, 405, 554

Allen, W. H. et al., 1993b, Astropart. Phys., 1, 269

Amenomori, M. et al., 2000, ApJ, 532, 302

Beck, R., 2008, in AIP Conf. Proc., High Energy Gamma-Ray Astronomy, 1085, 83

Bellido, J. A. (Pierre Auger Collaboration), 2005, in Proc. 29th ICRC, Pune, 00, 101

Bellido, J. A. (Pierre Auger Collaboration), 2009, in Proc. 31st ICRC, Łódź

Bellido, J. A., Clay, R. W., Dawson, B. R. \& Johnston-Hollitt, M., 2001, Astropart. Phys., 15, 167

Berezinskii, V. S., Bulanov, S. V., Dogiel, V. A., Ginzburg, V. L. \& Ptuskin, V. S., 1990, Astrophysics of Cosmic Rays (Amsterdam: North Holland)

Bird, D. J. et al., 1995, ApJ, 441, 144

Bird, D. J. \& Clay, R. W., 1990, PASA, 8, 266

Bond, I. A. et al., 1996, A\&A, 307, 708

Bonifazi, C. (Pierre Auger Collaboration), 2009, Nucl. Phys. B (Proc. Suppl.), 190, 20

Brown, A. M., 2009, in Proc. 5th DSU Conf., Melbourne 2009, arXiv:0908.1035

Carramiñana, A. et al., 1990, A\&A, 228, 327

Chadwick, P. M., Lyons, K., McComb, T. J. L., Orford, K. J., Osborne, J. L., Rayner, S. M., Shaw, S. E. \& Turver, K. E., 1999, ApJ, 521, 547

Clarke, T. E., Kronberg, P. P. \& Bohringer, H., 2001, ApJ, 547, L111

Clay, R. W., 2000, PASA, 17, 212

Clay, R. W., Gerhardy, P. R. \& Liebing, D. F., 1984, Aust. J. Phys., 37,91

Clay, R. W., Dawson, B. R. \& Meyhandan, R., 1994, Astropart. Phys., 2, 347

Di Giulio, C. (Pierre Auger Collaboration), 2009, in Proc. 31st ICRC, Łódź

Driver, S. P. et al., 2008, ApJ, 678, L101

D'Urso, D. (Pierre Auger Collaboration), 2009, in Proc. 31st ICRC, Łódź

Farley, F. J. M. \& Storey, J. R., 1954, Proc. Phys. Soc. Lond. A, 67,996

Feretti, L. \& Johnston-Hollitt, M., 2004, New Astron. Rev., 48, 1145
Gaisser, T. K. \& Stanev, T., 2006, Nucl. Phys. A, 777, 98

Grindlay, J. E., Helmken, H. F., Brown, R. H., Davis, J. \& Allen, L. R., 1975a, ApJ, 197, L9

Grindlay, J. E., Helmken, H. F., Brown, R. H., Davis, J. \& Allen, L. R., 1975b, ApJ, 201, 82

Grindlay, J. E., 1975, ApJ, 199, 49

Hanbury Brown, R., Davis, J. \& Allen, L. R., 1969, MNRAS 146,399

Hague, J. D. (Pierre Auger Collaboration), 2009, in Proc. 31st ICRC, Łódź

Hardcastle, M. J. et al., 2007, ApJ, 670, L81

Harton, J. L. (Pierre Auger Collaboration), 2009, in Proc. 31st ICRC, Łódź

Hillas, A. M., 1984, ARA\&A, 22, 425

Hillas, A. M., 2009, Astropart. Phys., 32, 160

James, C. W. et al., 2009, Nucl. Instr. Meth. Phys. Res. A, 604, S112

Kabuki, S. et al., 2007, ApJ, 668, 968

Kamata, K. et al., 1968, Can. J. Phys., 46, S72

Levinson, A., 2000, Phys. Rev. Lett., 85, 912

Nagar, M. N. \& Matulich, J., 2008, A\&A, 488, 879

Protheroe, R. J., 1986a, MNRAS, 221, 769

Protheroe, R. J., 1986b, PASA, 6, 280

Protheroe, R. J. \& Clay, R. W., 2004, PASA, 21, 1

Rieger, F. M. \& Aharonian, F. A., 2009, A\&A, in press (arXiv:0910.2327)

Rowell, G. P. et al., 1999, Astropart. Phys., 11, 217

Rowell, G. P., Stamatescu, V., Clay, R. W., Dawson, B. R., Protheroe, R. J., Smith, A. G. K., Thornton, G. J. \& Wild, N., 2008, Nucl. Instr. Meth. Phys. Res. A, 588, 48

Schüssler, F. (Pierre Auger Collaboration), 2009, in Proc. 31st ICRC, Łódź

Sreekumar, P., Bertsch, D. L., Hartman, R. C., Nolan, P. L. \& Thompson, D. J., 1999, Astropart. Phys., 11, 221

Stanev, T., 1997, ApJ, 479, 290

Steinle, H. et al., 1998, A\&A, 330, 97

Steinle, H., 2006, ChJAA Supp. 6, no. 1, 106

SUGAR Catalogue, 1986, Catalogue of Highest Energy Cosmic Rays No.2 SUGAR, World Data Center C2 for Cosmic Rays, Institute of Physical and Chemical Research, Itabashi, Tokyo

Sun, X. H. et al., 2008, A\&A, 477, 573

Suomijärvi, T. (Pierre Auger Collaboration), 2009, in Proc. 31st ICRC, Łódź

Turner, T. J., George, I. M., Mushotzky, R. F. \& Nandra, K., 1997, ApJ, 475, 118

Wahlberg, H. (Pierre Auger Collaboration), 2009, in Proc. 31st ICRC, Łódź

Winn, M. W. et al., 1986, J. Phys. G: Nucl. Phys., 12, 653 